\title{
The kinematic of HST-1 in the jet of M 87
}

\author{
Kazuhiro Hada*1, Marcello Giroletti ${ }^{1}$, Gabriele Giovannini ${ }^{1}$, Carolina Casadio ${ }^{2}$, \\ Matthias Beilicke ${ }^{3}$, Andrea Cesarini ${ }^{4}$, Teddy Cheung ${ }^{5}$, Akihiro Doi ${ }^{6}$, Henric \\ Krawczynski $^{3}$, Motoki Kino ${ }^{7}$, Nick Lee ${ }^{8}$, Hiroshi Nagai ${ }^{7}$
}

${ }^{1}$ INAF Istituto di Radioastronomia, Via Gobetti 101, 40129 Bologna, Italy

${ }^{2}$ Instituto de Astrofisica de Andalucia, CSIC, Apartado 3004, 18080 Granada, Spain

${ }^{3}$ Washington University, St. Louis, MO 63130, USA

${ }^{4}$ National University of Ireland, University Road, Galway, Ireland

${ }^{5}$ Space Science Division, Naval Research Laboratory, Washington, DC 20375-5352, USA

${ }^{6}$ Institute of Space and Astronautical Science, 3-1-1 Yoshinodai, Sagamihara, 229-8510

Kanagawa, Japan

${ }^{7}$ National Astronomical Observatory of Japan, 2-21-1 Osawa, Mitaka, 181-8588 Tokyo, Japan

${ }^{8}$ Smithsonian Astrophysical Observatory, 60 Garden St., Cambridge MA 02138, USA

E-mail: hada@ira.inaf.it

M 87 is a well-known radio galaxy showing intense gamma-ray emission up to Very-High-Energy (VHE) regime. Its proximity provides a unique opportunity to probe the connection of VHE gamma-ray production with relativistic jets in detail. Over recent years, the peculiar knot HST-1 has attracted a great interest as a possible site for the VHE gamma-ray production because of its violent behavior seen from radio to X-ray. To investigate its structural variation and a possible connection with the gamma-ray emission, we analyzed and compared 26 VLBI observations of the M 87 jet, obtained between 2006 and 2011 with the Very Long Baseline Array (VLBA) at $1.7 \mathrm{GHz}$ and the European VLBI Network (EVN) at $5 \mathrm{GHz}$. We successfully detected HST-1 at all epochs; we model-fitted its complex structure with two or more components, the two outermost of which display a significant proper motion with a superluminal velocity around $\sim 4 c$. We also found the emergence of a new feature from HST-1 upstream edge in the later epochs of 2010. While the structure has moved by over 80 mas downstream during the time of our observations, the overall position angle of HST-1 has changed from -65 to -90 degrees. These results on the component evolution suggest that structural changes at the upstream edge of HST-1 can be related to the VHE events.

11th European VLBI Network Symposium \& Users Meeting

9-12 October 2012

Bordeaux (France)

\footnotetext{
* Speaker.
} 


\section{Introduction}

The debate about the location and the mechanisms for the production of $\mathrm{MeV} / \mathrm{GeV}$, and very high energy (VHE) $\gamma$-rays in active galactic nuclei (AGN) jets is still very lively in the era of the Fermi satellite and the new generation Cherenkov telescopes. M 87 is a privileged laboratory for a detailed study of the properties of jets because of its proximity $(D=16 \mathrm{Mpc})$ with the massive black hole $\left(M_{\mathrm{BH}}=6.4 \times 10^{9} M_{\odot}\right.$, [1], leading to a scale of 1 mas $\left.\sim 140 R_{\mathrm{S}}\right)$. This source shows a prominent radio, optical, and X-ray jet, characterized by many substructures and knots from sub-parsec to kiloparsec scale. Closer to the jet base, VLBI observations show a well-resolved, edge-brightened jet structure with a broadening of the opening angle ([2, 3, 4, 5, 6, 7]), indicating that the collimation region is beginning to be resolved.

At about $0.8-0.9$ arcsec from the core, the jet suddenly rebrightens. This feature was first discussed in the optical band ([8]) and named as HST-1. It appeared to emit superluminal optical features with velocity $\sim 6 c$. Superluminal components within HST-1 were later found with much finer angular resolution thanks to VLBI observations at $1.7 \mathrm{GHz}$ ([9]). In addition to presenting this hallmark of blazar activity, HST-1 underwent a dramatic brightening in radio, optical, and X-rays during 2003-2006, becoming even brighter than the nucleus in X-rays ([10]). These facts together led Cheung et al. [9] to propose that the flaring activity registered at VHE in 2005 ([11]) originated in HST-1. On the other hand, the VHE variability on time scales of days seemed to require a much more compact emission region, suggesting the nucleus of M 87 itself as a likely site of $\mathrm{TeV} \gamma$-ray production ([11]). Indeed, a second VHE flare was observed in 2008, simultaneously to a strong increase of the $43 \mathrm{GHz}$ flux density of the core, while HST-1 was in a low state ([12]). Since these two candidates are located in remarkably different enviroments, the identification of the exact $\gamma$-ray emtting site in M 87 will affect significantly our understanding of $\gamma$-ray production processes.

In this context, we started at the end of 2009 a program to monitor M 87 at $5 \mathrm{GHz}$ with the European VLBI Network in real-time mode (e-EVN, [13]) during the season of VHE observations in 2009/2010. The chosen array configuration provides a suitable combination of resolution, sensitivity, and field of view, which permits a detailed study of the behavior of both the core and HST-1. Here we briefly summarize our initial results of this project, in which we especially focus on the kinematic properties of HST-1. The full details of the data analysis, results, discussion and figures are described in our recent paper [14].

\section{Observations and Data Reduction}

We observed M 87 with the e-EVN at 13 epochs between June 2009 and October 2011. Observations typically lasted $4-8 \mathrm{~h}$, and the longest baselines were achieved from European stations to Shanghai and/or Arecibo. The data quality is overall adequate to warrant good signal-to-noise $(\mathrm{S} / \mathrm{N})$ detections of the source structure. For all observations, the frequency setup was centered at $5.013 \mathrm{GHz}$ and divided into eight sub-bands separated by $16 \mathrm{MHz}$ each for an aggregate bit rate of 1 Gbps. The data were correlated in real time at JIVE, except for the first observation, which was disk-recorded; automated data flagging and initial amplitude and phase calibration were also carried out at JIVE using dedicated pipeline scripts. The data were finally averaged in frequency within each IF, but individual IFs were kept separate to avoid bandwidth smearing. Similarly, the 
data were time-averaged only to $8 \mathrm{~s}$ to avoid time smearing. In addition, we extend back to the past by analyzing 13 VLBA archival data at 1.7 or $2.3 \mathrm{GHz}$ obtained since 2006 November. The initial data calibration of the VLBA data was performed in NRAO AIPS. Final images (both EVN and VLBA) were produced after several cycles of phase and amplitude self-calibration. The final resolution and sensitivity of the VLBA and EVN data sets are well-matched (beam size in the range 5-10 mas and $\mathrm{rms}$ noise of $0.1-0.3 \mathrm{mJy} / \mathrm{beam})$.

\section{Results}

We detect significant flux density in the HST-1 region at all epochs, in addition to the bright core and the inner jet. The details of the imaging somewhat change depending on the observing frequency, the observation epoch, the $(u, v)$-plane coverage, and the adopted weighting scheme. The HST-1 region extends for over 50 mas and is resolved in complex substructures. The overall position angle and the location of the individual substructures evolve with time. To describe the emission from HST-1, we model-fitted the visibility data in Difmap for all epochs, adopting typically two or three elliptical Gaussian components. Fig. 1 shows the time evolution of the HST-1 region with ten contour images, overlaid with model-fit components. In terms of the kinematic properties of HST-1, we found the following remarkable facts during this period:

\section{- Significant proper motions over 5 years}

Thanks to the many observations and the good accuracy in fitting the structure, we can reliably track the components between the various epochs (see also Fig. 2 in [14]). In particular, the identification of the two main components are significant, which we label as component 1 and 2, of which 1 is the outermost. These have moved by very similar distances over 5 years between 2006.86 and $2011.80\left(\Delta r_{1}=88.1\right.$ mas, $\Delta r_{2}=81.2$ mas), corresponding to apparently superluminal velocities around $\beta_{\text {app }}=4.1$. The uncertainty on this superluminal value can be constrained down to as small as a few percent thanks to the number of observations.

\section{- Change of P.A.}

At the early epochs, an overall position angle of the HST-1 region is oriented similarly to the main jet of $\mathrm{M} 87\left(\sim-65^{\circ}\right)$. In the following epochs, the position angle progressively rotates to $\sim-90^{\circ}$ and finally to $\sim-100^{\circ}$ as the components move outward (see also Fig. 3 in [14]).

\section{- Structural variation and a new component emergence}

The size of each component varies and additional components are present at some epochs. In particular, component 2 becomes quite extended in early 2008 and eventually splits into two components from 2008.62. After this split, the upstream subcomponent remains more or less stationary and gradually becomes fainter. When the $5 \mathrm{GHz}$ observations start, there is little evidence of this component. However, starting from 2010.45, a new inner component is again required to fit the $5 \mathrm{GHz}$ data. This component appears consistently thereafter and we name it component 3 . This substructure is also moving superluminally, although the uncertainty is larger because of the shorter time range. 


\section{Discussion and Summary}

The observations presented here clearly demonstrate that HST-1 is resolved in complex substructures. Two main components can be reliably identified across epochs and they are found to move with superluminal velocities $(\sim 4 c)$. From this, we can infer a range of the possible intrinsic jet velocity, assuming that the pattern and bulk velocity are the same. Adopting a jet orientation angle in the range $15^{\circ}<\theta<25^{\circ}$ (e.g. [12]), the measured apparent velocity corresponds to an intrinsic velocity $0.97 c<v<0.99 c$, which in turn implies a Doppler factor and a Lorentz factor for this structure between $\delta_{\mathrm{HST}-1}=1.5$ and $\Gamma_{\mathrm{HST}-1}=6.5$ (for $\theta=25^{\circ}$ ), and $\delta_{\mathrm{HST}-1}=3.9$ and $\Gamma_{\mathrm{HST}-1}=4.1$ (for $\theta=15^{\circ}$ ), respectively. This result agrees with the synchrotron model for the X-ray emission discussed by Marshall et al. [15] and Harris et al. [16]. By contrast, different values of the apparent velocity have been reported by other authors in other parts of the jet and/or using data obtained in different epochs $([4,5,8,9,17])$, suggesting that the velocity structury jet is quite complex.

At first sight, our result does not support the identification of HST-1 as a standing-shock structure, given the displacements of $>80$ mas found for components 1 and 2 over $\sim 5$ years. Moreover, no prominent stationary components were consistently detected in the 26 observations considered in this work. However, the components change in size over time, and in particular component 2 splits into substructures after 2008.62, suggesting that other features exist in HST-1 and interact with the brightest knots. Those components may be underlying, standing or very slowly moving regions, too faint to be detected separately but contributing to the total emission when brighter components are nearby. In particular, this could be the case for the D component in Cheung et al. [9], which only becomes visible when a new feature (like our component 3 ) is ejected/created within HST-1. Such a behavior would then support the scenario in which HST-1 is a stationary reconfinement shock structure, possibly associated to a jet interaction with a gaseous condensation of the hot interstellar medium [18].

In Fig. 1 we show the epochs of the two latest VHE flares from M 87 with long dashed lines. Interestingly, both events are followed by structural changes and rebrightening of the upstream edge of HST-1 (see also Fig. 2 in [14]), suggesting that the origin of the VHE activity could indeed be related to the HST-1 region. A similar connection was also put forward for the 2005 VHE event: by considering a subset of the present dataset and archival VLA observations, a change in the proper motion velocity in HST-1 at the epoch $\sim 2005.5$ is suggested [19], coincident with the TeV $\gamma$-ray activity and the maximum radio and X-ray flux density of the feature.

We keep in mind, however, that the recent investigations of the M 87 jet base near the epoch of the VHE flare in April 2010 suggests a slight flux variation of the radio core [20] together with an enhanced X-ray core flux $([21,22])$. More investigations are thus needed to locate the exact $\gamma$-ray production sites unambiguously. Our deep e-EVN observations of M 87 are still actively ongoing. Moreover, we have recently started a dense monitoring program (almost biweekly) of several bright $\gamma$-ray sources including M 87 with VERA at $22 \mathrm{GHz}$ [23], which permits a detailed study of the core region. The combination of these monitorings will be powerful to understand the connection of $\gamma$-ray production with the M 87 jet.

Acknowledgments. We acknowledge a contribution from the Italian Foreign Affair Minister under 
the bilateral scientific collaboration between Italy and Japan. e-VLBI research infrastructure in Europe is supported by the European Union's Seventh Framework Programme (FP7/2007-2013) under grant agreement no. RI-261525 NEXPReS. The European VLBI Network is a joint facility of European, Chinese, South African and other radio astronomy institutes funded by their national research councils. The National Radio Astronomy Observatory is a facility of the National Science Foundation operated under cooperative agreement by AUI.

\section{References}

[1] Gebhardt, K., \& Thomas, J. 2009, ApJ, 700, 1690

[2] Junor, W., Biretta, J. A., \& Livio, M. 1999, Nature, 401, 891

[3] Krichbaum, T. P., Zensus, J. A., \& Witzel, A. 2005, AN, 326, 548

[4] Ly, C., Walker, R. C., \& Junor, W. 2007, ApJ, 660, 200

[5] Kovalev, Y. Y., Lister, M. L., Homan, D. C., \& Kellermann, K. I. 2007, ApJ, 668, L27

[6] Hada, K., Doi, A., Kino, M., et al. 2011, Nature, 477, 185

[7] Asada, K., \& Nakamura, M. 2012, ApJ, 745, L28

[8] Biretta, J. A., Sparks, W. B., \& Macchetto, F. 1999, ApJ, 520, 621

[9] Cheung, C. C., Harris, D. E., \& Stawarz, Ł. 2007, ApJ, 663, L65

[10] Harris, D. E., Cheung, C. C., Biretta, J. A., et al. 2006, ApJ, 640, 211

[11] Aharonian, F.,Akhperjanian, A.G.,Bazer-Bachi, A. R., et al. 2006, Science, 314, 1424

[12] Acciari, V. A., Aliu, E., Arlen, T., et al. 2009, Science, 325, 444

[13] Szomoru, A. 2008, Proc. Science, PoS (IX EVN Symp.) 040

[14] Giroletti, M., Hada, K., Giovannini, G., et al. 2012, A\&A, 538, L10

[15] Marshall, H. L., Miller, B. P., Davis, D. S., et al. 2002, ApJ, 564, 683

[16] Harris, D. E., Biretta, J. A., Junor, W., et al. 2003, ApJ, 586, L41

[17] Asada, K., Nakamura, M., Doi, A., Nagai, H., \& Inoue, M. 2011, IAUS, 275, 198

[18] Stawarz, Ł., Aharonian, F., Kataoka, J., et al. 2006, MNRAS, 370, 981

[19] Giovannini, G., Casadio, C., Giroletti, M., Beilicke, M., Cesarini, A., \& Krawczynski, H. 2011, IAUS, 275,150

[20] Hada, K., Kino, M., Nagai, H., et al. 2012, ApJ, 760, 52

[21] Abramowski, A., Acero, F., Aharonian, F., et al. 2012, ApJ, 746, 151

[22] Harris, D. E., Massaro, F., Cheung, C. C., et al. 2011, ApJ, 743, 177

[23] Nagai, H., Kino, M., Niinuma, K., et al. 2012, [arXiv:1210.2496] 


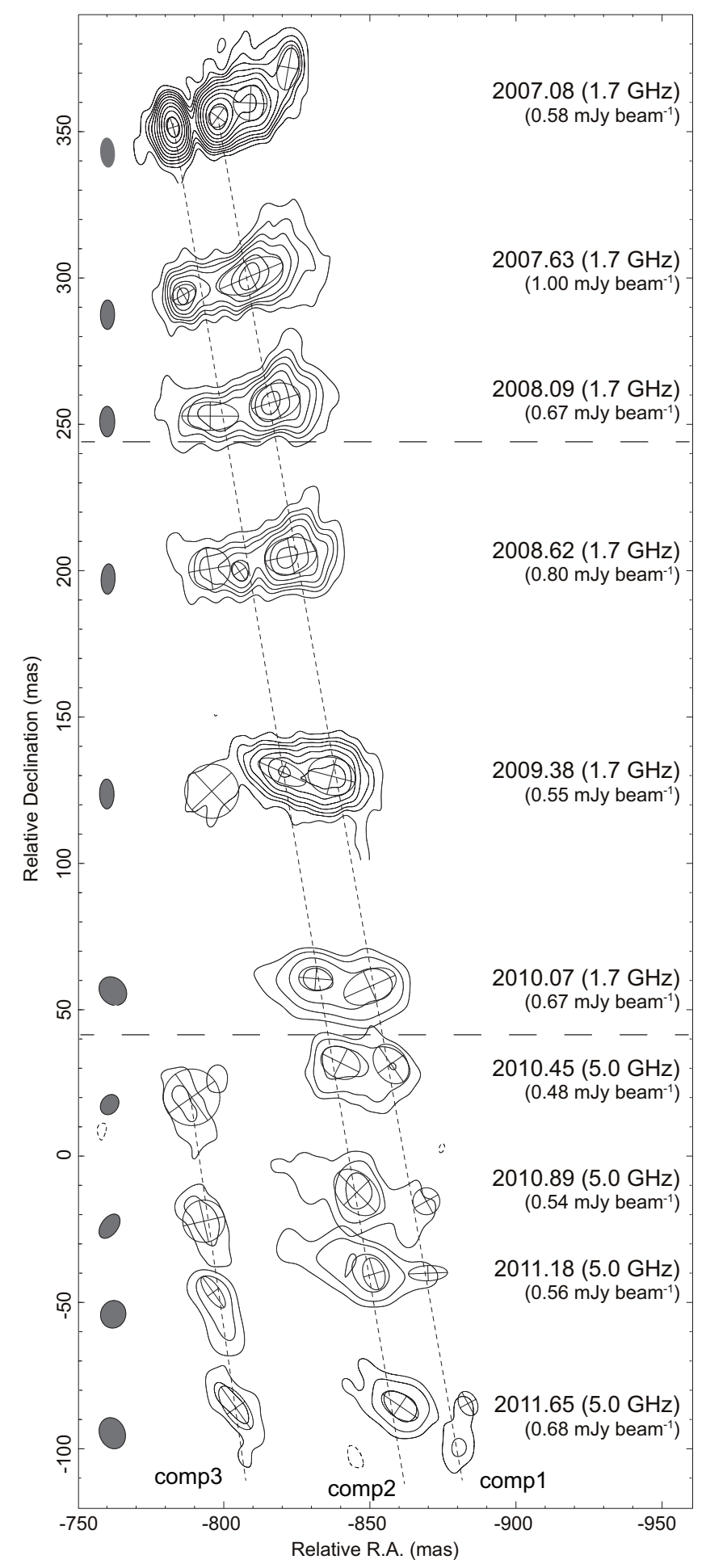

Figure 1: Set of HST-1 images. For each epoch, we give the epoch, frequency, lowest contour to the right, and the restoring beam to the left of the respective contour plot. Contours are traced at $(-1,1,2,4 \ldots)$ multiples of the given value. The model-fit components are overlaid as ellipses with crosses. The contour plots are spaced vertically proportionally to the time interval between the relative epochs. Two horizontal long-dashed lines indicate the epochs of the recent VHE $\gamma$-ray flares in 2008 and 2010. The axes represent the relative (RA, Dec) coordinates from the core for the first image. 